\title{
Efficacy and Safety of Scopolamine Compared to Placebo in Individuals with Bipolar Disorder Who are Experiencing a Depressive Episode (SCOPE-BD): Study Protocol for a Randomized Double-Blind Placebo-Controlled Trial
}

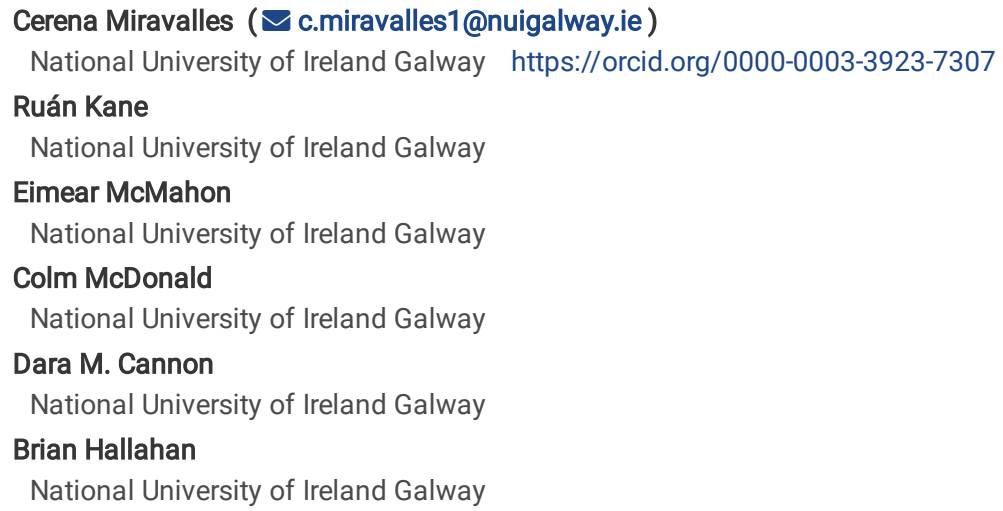

\section{Research Article}

Keywords: Bipolar disorder, scopolamine, depressive episode, cholinergic, muscarinic, antidepressant

Posted Date: March 7th, 2022

DOI: https://doi.org/10.21203/rs.3.rs-1315870/v1

License: (c) (i) This work is licensed under a Creative Commons Attribution 4.0 International License. Read Full License 


\section{Abstract}

\section{Background:}

Current treatment options for the management of depressive episodes in bipolar disorder are often sub-optimal, with some treatments either noted to be only partially effective or to require long durations of treatment prior to a therapeutic response. Therefore, pharmaco-therapeutic options that reduce depressive symptoms in a more rapid manner, might provide a viable therapeutic option for some people. Intravenous (IV) Scopolamine, a pan muscarinic antagonist has been demonstrated in a number of studies to confer a rapid antidepressant effect, albeit no study to date has exclusively evaluated its potential therapeutic effect in a cohort consisting solely of individuals with bipolar disorder.

\section{Methods:}

Individuals with bipolar disorder who are currently experiencing a depressive episode of at least moderate severity will be included in this study. Eligible participants will undergo a screening and placebo-run in visit, and will be randomised at visit 3 to the treatment or placebo group. Participants will receive the three blinded infusions over the course of two weeks, with two subsequent follow-up visits, one and three weeks after the last infusion visit. The total duration of the study will be approximately six weeks. Patients will continue their regular treatment regime in addition to study medication. Objective and subjective mood questionnaires, cognitive assessments and other psychometric instruments will be administered and recorded.

\section{Discussion:}

To our knowledge, this is the first study to investigate the antidepressant effects of IV scopolamine in an exclusively bipolar disorder cohort. Trial findings will contribute to the evidence base regarding the cholinergic hypothesis of mood disorders and specifically might result in an additional safe therapeutic option for the management of depressive episodes in bipolar disorder.

\section{Trial registration:}

ClinicalTrials.gov: NCT04211961

EudraCT Number: 2017-003112-39

\section{Background}

Bipolar disorder is a chronic disabling psychiatric disorder characterized by recurrent episodes of mania or hypomania and depression. Individuals with bipolar I disorder experience episodes of mania and depression, while individuals with bipolar II disorder characterizes experience episodes of depression with periods of hypomania, but not mania [1]. Bipolar disorder has an estimated prevalence of approximately $1 \%$ and a roughly equal gender ratio [2].

Current pharmacological treatments for depressive episodes in bipolar disorder remain sub-optimal, with pharmacological strategies employed to date often only partially effective $[3,4]$. A number of recent studies have suggested that scopolamine, a pan muscarinic(M) receptor antagonist can elicit a rapid antidepressant response in both major depressive disorder (MDD) and bipolar disorder [5-7] and thus may present a novel therapeutic strategy, particularly for the management of bipolar disorder, in individuals experiencing depressive episodes [8].

A rapid-acting anti-depressant effect has been demonstrated in some double-blind placebo-controlled trials of intravenous (IV) scopolamine in MDD or bipolar disorder $[6,7,9,10]$, but these findings have not been universally reported with other trials not reporting a significant treatment effect [11, 12]. Some studies have also examined, other modes of scopolamine administration. For example, a previous study of intramuscular scopolamine demonstrated no antidepressant effect [11]. A randomised controlled trial (RCT) of oral scopolamine as an augmentation agent demonstrated an antidepressant effect; however, this effect was not rapid-acting and the patient cohort did not include individuals with bipolar disorder [13]. RCTs relating to scopolamine administration utilising a transdermal patch for the management of depressive episodes have yet to be published. Thus, studies of IV scopolamine as a treatment agent for depressive episodes whilst promising in relation to their potential therapeutic effect, have to date included limited numbers of participants and diagnostically and clinically heterogeneous populations. This study is consequently significant as it will be the first RCT to date, to exclusively examine depressive episodes in bipolar disorder to ascertain if a rapid antidepressant effect is demonstrated.

Scopolamine, also known as "hyoscine" is most commonly utilised for the management of post-operative nausea, motion sickness and hypersalivation secondary to psychotropic medications such as clozapine [14-16]. Scopolamine acts as an inhibitor at postganglionic muscarinic cholinergic receptor sites in the parasympathetic nervous system. Muscarinic cholinergic receptors (CHRMs), which recognise the neurotransmitter, acetylcholine (ACh), are a family of seven-transmembrane domain receptors consisting of five receptor subtypes (M1-5) and are associated with heterotrimeric G-proteins which translate a transduction cascade $[17,18]$.

The cholinergic system has long been implicated as a contributory factor in the aetiology of mood disorders. Physostigmine, a cholinesterase inhibitor (opposite effect to scopolamine) has been demonstrated to exacerbate depressive symptomatology [19, 20] and rescue deficits in emotion inhibition [21] in individuals with bipolar disorder. These observations have led to the hypothesis that an imbalance between central cholinergic and adrenergic neurotransmitter activity could induce manic and depressive episodes [20]. Both, over-activity and oversensitivity of the cholinergic system have been associated with depressive symptomatology, with a reduction in $\mathrm{M} 2$ receptors in vivo noted during depressive episodes [22], which is partially explained by variation in the M2 gene [23]. M2-receptor antagonism by scopolamine may potentially regulate activity of this system and therefore reduce the oversensitivity associated with depressive episodes. Consequently, evidence of a putative rapid antidepressant effect with IV scopolamine infusions may be 
of particular benefit for individuals with bipolar disorder who are experiencing depressive episodes. IV scopolamine could thus present an additional pharmacological strategy for the management of clinical and cognitive signs, symptoms and deficits in bipolar disorder by alleviating depressive symptomatology and ameliorating patient functioning.

The aim of this clinical trial is to investigate the efficacy and safety of IV Scopolamine, compared to placebo, in reducing the severity of depressive symptoms in individuals with bipolar disorder who are experiencing a depressive episode of at least moderate severity. In addition, the study will investigate if IV Scopolamine impacts cognition and improves functioning as measured on a number of objective and subjective psychometric instruments when compared to placebo.

\section{Methods}

\section{Study Design}

This is a single-site, randomised, double-blind, placebo-controlled, parallel, phase Ilb clinical trial examining if IV scopolamine exhibits an antidepressant effect in individuals with bipolar disorder. Study visits will take place at the Clinical Research Facility Galway (CRFG), in University Hospital Galway, Galway, Ireland. Patients will be recruited from mental health services in the West of Ireland including a specialised bipolar disorder clinic at University Hospital Galway.

Individuals will have a diagnosis of bipolar disorder according to the Diagnostic Statistics Manual (DSM)-V and must be experiencing a depressive episode of at least moderate severity based on a clinical interview by a trained clinician and a Hamilton Depression Rating Scale (HDRS) score $\geq 14$. Exclusion criteria include being euthymic or experiencing a manic or hypomanic episode; treatment with any cholinergic medications (i.e. biperiden, procyclidine); a history of a previous allergic reaction or sensitivity to scopolamine, treatment with oral steroids, a history of an alcohol or substance use disorder in the three months prior to study entry, an intellectual disability (intelligence quotient $<70$ ), dementia or cognitive impairment and severity of depressive episode so that participation in a clinical trial is not appropriate.

Patients meeting all inclusion criteria will be enrolled into the study (duration $\sim 6$ weeks total). The maximum time between Visit 1 (screening) and Visit 2 is 14 days. The screening visit and Visit 2 can occur on the same day. At Visit 2, all participants will receive a placebo run-in (100 ml of Saline IV). This has been utilised in other IV Scopolamine RCTs [5-7, 9] and has the advantage of reducing a subsequent placebo effect (which might result in a Type II error) and a lower loss to follow-up rate post-randomisation $[19,24]$.

Within 7 days of Visit 2, participants will be assessed against the placebo run-in criteria and if these criteria are met (HDRS score $\geq 8$ at Visit 3 ), they will be randomised to receive either placebo $(n=25)$ or $4 \mu \mathrm{g} / \mathrm{kg}$ Scopolamine $(n=25)$ IV in $100 \mathrm{ml}$ of Saline over 15 minutes at:

- Visit 3 (Day 0),

- Visit 4 (days 2-6) and

- Visit 5 (days 6-10)

Participants will receive Scopolamine or placebo in addition to their current treatment regimen. At least two days will elapse between IV infusions (Visits 2-5). Two follow-up visits (Visits 6 and 7) will occur at days 15 (+/-5 days) and days 29 (+/- 7 days) with at least two days between Visits 5 and the first follow-up visit (Visit 6) and at least three days between the two follow-up visits (Visits 6 and 7). If a trial participant requires medical intervention during or postinfusion, the treating physician must assess if it is appropriate to proceed with the current and/or future infusions. The flow of the study visits is presented in Figure 1.

\section{Note timeline between visits:}

Visit 1 and 2: $\leq 14$ days. Can also occur on the same day and thus data does from screening visit does not need to be replicated at Visit 2.

Visit 2 and $3: \geq 2$ days and $\leq 7$ days

Visit 3 and Visit 4 and Visit 5 and Visit $6: \geq 2$ days

Visit 6 and Visit 7: $\geq 3$ days

Psychometric Instruments

\section{Clinical Instruments}

\section{Alcohol Use Disorder Identification Test (AUDIT) [25].}

A 10-item questionnaire to identify harmful use or dependence on alcohol.

Clinical Global Impression (CGI)[26].

A rating scale that measures illness severity (CGI-S) (1-7), and global improvement (CGI-I) (1-7)) and has an efficacy index (which relates therapeutic effects to adverse effects) (1-4). 
Fagerstrom Test for Nicotine Dependence [27].

A 6-item questionnaire examining nicotine dependence.

Global Assessment of Functioning (GAF) [28].

A rating scale from 1-100 that measures an individual's functioning and is observer rated.

Hamilton Depression Rating Scale (HDRS) [29].

A 21-item observer rated instrument that measures symptoms of depression.

Montgomery and Asberg Depression Rating Scale (MADRS) [30].

A 10-item observer rated scale that measures symptoms of depression.

NEO Personality Inventory-Five Factor Inventory (NEO PI-FFI) [31].

This 60 -item personality inventory measures personality traits in five dimensions (neuroticism, extraversion, openness to experience, agreeableness and conscientiousness).

Patient Rated Inventory of Side Effects (PRISE) [32].

A 9-item self-report instrument measuring adverse effects associated with the gastrointestinal, genitourinary, nervous and cardiac systems, sense organs, skin, sexual functioning, sleep and other general adverse effects.

Profile of Mood State(POMS) [33].

A rating scale where 65 adjectives are rated by participants on a 5-point Likert scale. Six factors are derived from these adjectives (tension, depression, anger, fatigue, vigour and confusion).

\section{Structured Clinical Interview for DSM-5 Disorders (SCID-5-RV) [34].}

This is a semi-structured interview that evaluates pathology and includes a demographic section followed by nine diagnostic modules, including two that examine mood disorders.

\section{Visual Analog Scale(VAS).}

A self-report scale used to indicate on a 10-point Likert how participants feel they are on various items, with the VAS utilised for the following terms in this study: (1) happy, (2) restless, (3) sad, (4) anxious, (5) anger, (6) drowsiness and (7) alertness. These items are consistent with those used by Furey et al. (2012) in their study of Scopolamine in mood disorders.

Young Mania Rating Scale(YMRS) [35].

An 11-item observer rated instrument that measures symptoms of elation.

\section{Cognitive Instruments}

The Cambridge Neuropsychological Test Automated Battery (CANTAB) [36].

Tests conducted from this battery will entail: (1) Emotion Recognition Task (ERT) (assesses ability to identify emotions through facial expressions), (2) Paired Associates Learning (PAL) (assesses visual memory and new learning), (3) One Touch Stockings of Cambridge (OTC) (assess executive function through spatial planning and working memory), and (4) Rapid Visual Information Processing (RVP) (assesses attention).

\section{Wechsler Adult Intelligence Scale $3^{\text {rd }}$ Edition (WAIS-III) [37].}

Full scale, verbal and performance Intelligence Quotient (IQ) will be estimated using an abbreviated version of this instrument, and will comprise the verbal subscales of vocabulary and similarities, and the performance subscales of block-design and matrix reasoning.

See Table 1 for the schedule of these psychometric assessments.

Table 1. Schedule of Assessments 


\begin{tabular}{|c|c|c|c|c|c|c|c|c|c|c|c|c|c|c|c|}
\hline \multirow{6}{*}{ Procedures } & \multirow{6}{*}{$\begin{array}{l}\text { Visit } 1 \\
\text { Screening }\end{array}$} & \multicolumn{3}{|c|}{ Visit 2} & \multicolumn{3}{|c|}{ Visit 3} & \multicolumn{3}{|c|}{ Visit 4} & \multicolumn{3}{|c|}{ Visit 5} & \multirow{4}{*}{$\begin{array}{l}\text { Visit } 6 \\
\text { Day } 15 \\
( \pm 5 \\
\text { Days) } \\
\text { Follow- } \\
\text { Up }\end{array}$} & \multirow{4}{*}{$\begin{array}{l}\text { Visit } 7 \\
\text { Day } 29 \\
( \pm 7 \\
\text { Days) } \\
\text { Follow- } \\
\text { Up }\end{array}$} \\
\hline & & \multirow{3}{*}{\multicolumn{3}{|c|}{ PLACEBO }} & \multicolumn{6}{|c|}{ Randomisation } & & & & & \\
\hline & & & & & Day & & & Day & ( \pm 2 Days & & \multicolumn{3}{|c|}{ Day 8 ( \pm 2 Days) } & & \\
\hline & & & & & \multicolumn{3}{|c|}{$\begin{array}{l}\text { Scopolamine or } \\
\text { Placebo }\end{array}$} & \multicolumn{3}{|c|}{$\begin{array}{l}\text { Scopolamine or } \\
\text { Placebo }\end{array}$} & \multicolumn{3}{|c|}{$\begin{array}{l}\text { Scopolamine or } \\
\text { Placebo }\end{array}$} & & \\
\hline & & \multicolumn{3}{|c|}{ IV Infusion } & \multicolumn{3}{|c|}{ IV Infusion } & \multicolumn{3}{|c|}{ IV Infusion } & \multicolumn{3}{|c|}{ IV Infusion } & & \\
\hline & & Pre & During & Post & Pre & During & Post & Pre & During & Post & Pre & During & Post & & \\
\hline $\begin{array}{l}\text { Signed informed } \\
\text { consent }\end{array}$ & $x$ & & & & & & & & & & & & & & \\
\hline Inclusion/Exclusion & $\mathrm{x}$ & $\mathrm{x}$ & & & $\mathrm{x}$ & & & & & & & & & & \\
\hline Randomisation & & & & & $\mathrm{x}$ & & & & & & & & & & \\
\hline IWRS & & & & & $x$ & & & $x$ & & & $x$ & & & & \\
\hline Demography & $\mathrm{x}$ & & & & & & & & & & & & & & \\
\hline MEHI & $\mathrm{x}$ & & & & & & & & & & & & & & \\
\hline $\begin{array}{l}\text { Medical/Surgical } \\
\text { History/History } \\
\text { BPD }\end{array}$ & $x$ & & & & & & & & & & & & & & \\
\hline Current Medication & $x$ & $x$ & & & $x$ & & & $x$ & & & $x$ & & & $x$ & $\mathrm{x}$ \\
\hline $\begin{array}{l}\text { Vital Signs - HR, } \\
\text { BP \& RR }\end{array}$ & $x$ & $x$ & & $\mathrm{x}$ & $\mathrm{x}$ & & $x$ & $x$ & & $x$ & $\mathrm{x}$ & & $x$ & & \\
\hline SCID-RV & $x$ & & & & & & & & & & & & & & \\
\hline HDRS & $x$ & $\mathrm{x}$ & & & $\mathrm{x}$ & & & $\mathrm{x}$ & & & $x$ & & & $\mathrm{x}$ & $\mathrm{x}$ \\
\hline YMRS & $x$ & $x$ & & & $x$ & & & $x$ & & & $x$ & & & $x$ & $x$ \\
\hline AUDIT & $x$ & & & & & & & & & & & & & & \\
\hline $\begin{array}{l}\text { Pregnancy } \\
\text { Discussion }\end{array}$ & $x$ & $x$ & & & $\mathrm{x}$ & & & $x$ & & & $x$ & & & & \\
\hline $\begin{array}{l}\text { Contraception } \\
\text { Advice }\end{array}$ & $\mathrm{x}$ & $\mathrm{x}$ & & & $x$ & & & $\mathrm{x}$ & & & $x$ & & & & \\
\hline $\begin{array}{l}\text { Serum Pregnancy } \\
\text { Test }^{D}\end{array}$ & & $x$ & & & & & & & & & & & & & \\
\hline $\begin{array}{l}\text { Pregnancy urine } \\
\text { dipstick }^{\mathrm{D}}\end{array}$ & & $x$ & & & $x$ & & & $x$ & & & $\mathrm{x}$ & & & & \\
\hline U\&Es, LFTs, TFTs。 & $\mathrm{x}$ & $x$ & & & & & & & & & & & & & \\
\hline Fagerstrom & & $x$ & & & & & & & & & & & & & \\
\hline CGI-S & & $x$ & & & $x$ & & & $x$ & & & $x$ & & & $x$ & $x$ \\
\hline CGI-I & & & & & $x$ & & & $x$ & & & $x$ & & & $x$ & $x$ \\
\hline VAS & & $x$ & & $x$ & $x$ & & $x$ & $x$ & & $x$ & $x$ & & $x$ & & \\
\hline MADRS & & $x$ & & & $x$ & & & $x$ & & & $x$ & & & $x$ & $x$ \\
\hline GAF & & & & & $x$ & & & & & & $x$ & & & $x$ & $\mathrm{x}$ \\
\hline ECG & & $x$ & & & & & & & & & & & & & \\
\hline $\begin{array}{l}\text { Height }(\mathrm{cm}) \& \\
\text { Weight }(\mathrm{kg})\end{array}$ & & $x$ & & & & & & & & & & & & & \\
\hline IV Cannulation & & $x$ & & & $x$ & & & $x$ & & & $x$ & & & & \\
\hline $\begin{array}{l}\text { Infusion } \\
\text { administration }\end{array}$ & & & $x$ & & & $x$ & & & $x$ & & & $x$ & & & \\
\hline Adverse Events & & $x$ & $\mathrm{x}$ & $\mathrm{x}$ & $\mathrm{x}$ & $x$ & $x$ & $\mathrm{x}$ & $\mathrm{x}$ & $x$ & $x$ & $x$ & $x$ & $\mathrm{x}$ & $\mathrm{x}$ \\
\hline $\begin{array}{l}\text { Cannulation Site } \\
\text { Check }\end{array}$ & & $\mathrm{x}$ & & $\mathrm{x}$ & $x$ & & $x$ & $\mathrm{x}$ & & $\mathrm{x}$ & $x$ & & $x$ & & \\
\hline
\end{tabular}




\begin{tabular}{|c|c|c|c|c|c|c|c|c|c|c|c|}
\hline Proded(Desional) & Visit 1 & Xisit 2 & $x$ & Xisit 3 & $x$ & Xisit 4 & $x$ & Xisit 5 & $x$ & Xisit 6 & Xisit 7 \\
\hline CANTAB ** & \multirow{3}{*}{\multicolumn{2}{|c|}{ Screening }} & $x$ & & $x$ & & & & & $x$ & \multirow{4}{*}{$\begin{array}{l}\text { Day } 29 \\
X^{\star} \\
( \pm 7 \\
\text { Days) }\end{array}$} \\
\hline (Upturial) & & & & Randomisation & & & & & & \multirow{3}{*}{$\begin{array}{l}\text { Day } 15 \\
X^{\star} \\
( \pm 5 \\
\text { Rays })\end{array}$} & \\
\hline WAIS * (Optional) & & & $X^{*}$ & Day 0 & $X^{*}$ & Day 4 ( \pm 2 Days $)^{x}$ & $X^{*}$ & Day 8 ( \pm 2 Days $)^{?}$ & $X^{*}$ & & \\
\hline NEO-PI-FFI * & & PLACEBO & $X^{*}$ & Scopolamine or & $X^{*}$ & Scopolamine or & $X^{*}$ & Scopolamine or & $X^{*}$ & & \\
\hline PRISE (Optional) & & & $x$ & Placebo & $x$ & Placebo & $x$ & Placebo & $x$ & $\begin{array}{l}\text { Follow- } \\
\text { Up }\end{array}$ & $\begin{array}{l}\text { Follow } \\
\text { Up }\end{array}$ \\
\hline $\begin{array}{l}\text { Subjective } \\
\text { Assessment }\end{array}$ & & IV Infusion & & IV Infusion & & IV Infusion & & IV Infusion & & & $x$ \\
\hline
\end{tabular}

** These include the Emotion Recognition Task (ERT), Paired Associates Learning (PAL), One Touch Stockings of Cambridge (OTC), and Rapid Visual Information Processing (RVP) (assesses attention) tests.

D Pregnancy test (serum pregnancy test and pregnancy urine dipstick), when required, for female participants only. Serum result confirmed prior to Visit 3.

${ }^{\circ}$ Blood tests for U\&Es (Urine and Electrolytes), LFTs (Liver Function Tests) and TFTs (Thyroid Function Tests) should be confirmed within acceptable ranges in the previous 4 months of the Screening (Visit 1). Can be performed if required at Visit 1 or 2 (results must be confirmed as acceptable prior to infusion).

AUDIT = Alcohol Use Disorder Identification Test; BP = Blood Pressure; CANTAB = Cambridge Neuropsychological Test Automated Battery, CGI-I = Clinical Global Impression - Improvement, CGI-S= Clinical Global Impression- Severity, ECG= Electrocardiograph; Fagerstrom: Test for Nicotine Dependence, GAF = Global Assessment of Functioning; HDRS = Hamilton Depression Rating Scale; HR = Heart Rate; IV = Intravenous; IWRS = Interactive Web Response System; MADRS = Montgomery and Asberg Depression Rating Scale; MEHI = Modified Edinburgh Handedness Inventory; NEO PI-FFI = NEO Personality Inventory-Five Factor Inventory; POMS = Profile of Mood States; PRISE= Patient Rated Inventory of Side Effects; RR = Respiratory Rate; SCID-RV = Structured Clinical Interview for DSM; VAS = Visual Analogue Scale; YMRS = Young Mania Rating Scale; WAIS = Wechsler Adult Intelligence Scale.

\section{Data collection and randomisation}

Clinical interviews and psychometric assessments will be collected by trained clinicians and research associates with a background in psychiatric research. All research staff involved in administration of clinical instruments or data-input will have up-to-date training in Good Clinical Practice (GCP)[38]. Infusions will be dispensed by the pharmacy and prepared by unblinded nurses, all attached to the CRFG.

Participants will be randomly assigned to receive either scopolamine or placebo in a 1:1 ratio. Randomly permuted blocks of sizes 4 and 6 will be used to ensure similar numbers of participants in each arm of the trial. Randomisation will be stratified by the HDRS score at trial entry (a score of < 23 indicating a mild-moderate depressive episode and a score $\geq 23$ indicating a severe depressive episode). This will help ensure greater balance between arms in the final trial sample and increase the efficiency of our treatment effect estimates.

A validated randomisation system will be used at Visit 3, (after the HDRS and YMRS are completed) to randomise patients to either arm. This centralised system will ensure allocation concealment, preventing blinded trial staff from knowing which treatment group will be allocated. Blocks of randomly varying length will also reduce the predictability of the allocation sequence. Randomisation will be carried out by the pharmacy team. In the case of an emergency, when knowledge of the treatment assignment is essential for the clinical management of the participant, a treating physician may request to unblind a single participant.

\section{Treatments}

\section{Treatment group}

Participants randomised to the scopolamine group will receive a 15-minute infusion of IV Scopolamine in 100 ml saline, repeated over 3 visits (Visit 3, 4 \& 5).

\section{Placebo group}

Participants randomised to the placebo group will receive a 15-minute infusion of IV saline, repeated over 3 visits (Visit 3, 4 \& 5).

Non-adherence will be extremely unlikely given the mode of administration, with participants monitored by a team researcher throughout the infusion period.

\section{Sample size calculations}

Based on previous data [9], the allocation of 22 participants to each arm will ensure a power of $\geq 85 \%$ to detect a $50 \%$ reduction (treatment response) on the HDRS in the scopolamine compared to the placebo group. The sample size is based on a 2-sample t-test with a conservative standard deviation of 13 units and a significance level of 0.05 . Allocating 25 participants to each arm will allow a loss to follow-up of approximately $12 \%$ post-randomisation (6 participants missing at Visit 6 follow-up). It is suggested that approximately 60 participants will need to be recruited to ensure 50 participants (83.3\%) are eligible for randomisation (Visit 3). All participants will attain a placebo infusion at Visit 2.

\section{Safety monitoring}


Adverse events (AE's) and serious adverse events (SAE's) will be recorded on AE case report forms for all participants from the time of consent for the duration of their participation in the study. A full AE review will be conducted at each study visit to ensure that a complete evaluation of the safety and tolerability of the investigational medical product (placebo or scopolamine) is conducted. The site investigator will follow-up all AE's reported during the treatment period until resolved, considered stable, including completion of patient participation in the trial (i.e. final follow-up visit). All SAE's will be followedup until resolution or until they are clearly determined to be due to a participant's stable or chronic condition or intercurrent illness(es) including after trial completion if required. SAEs will be reported to the sponsor and principal investigator within 24 hours after site awareness of the SAE to find a reasonable solution. Any suspected unexpected serious adverse reaction (SUSAR) that potentially occur will be reported by the sponsor to the competent authority (HPRA and/or EudraVigilance), the approving ethics committee and PI. Indemnity is in place pertaining to any adverse effects experienced by study participants.

\section{Statistics}

\section{Primary effect analysis}

The mean scores at Visit 6 will be compared across the study arms using an analysis of co-variance (ANCOVA) model. The response variable will be the change in HDRS score (primary outcome measure) from Visit 3 to Visit 6. For this effect analysis of the primary outcome, HDRS score at Visit 3 is added as a co-variate as it is expected that pre-randomisation measurements of the HDRS score will be correlated with scores obtained at Visit 6 . The inclusion of stratifying variables (i.e. severe v. mild or moderate depressive symptoms at baseline) and other variables as covariates (i.e. bipolar I or II disorder) will be considered as appropriate to increase the power to detect significant differences between the groups. Inverse probability weighting will be applied to the primary outcome analysis. These weights will be derived based on the inverse of the probability of a patient's data being missing given their prerandomisation measurements. This will ensure the estimate and inference is more representative of all patients randomised, reducing bias in the estimation of the treatment effect due to participants being lost to follow-up and missing data. Inference regarding the treatment effectiveness will focus on the point estimate, confidence interval and p-value for hypothesis confirmation.

\section{Secondary effect analysis}

Secondary outcomes will be analysed as appropriate (according to the distribution of each outcome), comparing the difference between groups at the specified follow-up visit and relative to baseline values (where specified/measured). Baseline variables predictive of each outcome (the measure at baseline where available), and stratifying variables will be included in ANCOVA or generalised linear models of outcome variables. For secondary outcomes (i.e. acute and long-term effects on cognition (CANTAB), change in other mood rating scales including the MADRS and POMS, changes in functioning (GAF), treatment changes), the focus will be on the point estimates and confidence intervals for hypothesis generation.

Additional analysis of the primary outcome will compare the time course of HDRS under placebo and treatment using a mixed-effects model including fixedeffects terms for time since randomisation/study visit, trial arm and their interactions, and a random effect to account for correlation of multiple observations per participant.

\section{Ethical approval}

Required documents including the study protocol (V3.0, $18^{\text {th }}$ December, 2020), informed consent form, participant information leaflet, and any other required documents (i.e. psychometric instruments) have been submitted and approved by the Galway University Hospitals Research Ethics Committee and the Health Products Regulatory Authority (HPRA). The sponsor (Research Office, NUI Galway) will submit and obtain approval from the above parties for substantial amendments to the original approved documents.

\section{Data management and monitoring}

All study information will be stored in Microsoft Excel 365 by a data manager using an electronic database. The database will record all subject data to include the baseline characteristics, pre- and post-assessments, and possible adverse events. The computer and database will use password protection with the database only accessible to the study researchers. To ensure the confidentiality of the data, all subjects will be provided with identification numbers. All researchers will have access to the final trial data. Electronic data and paper documents will be kept for 5 years, after which they will be destroyed.

A Data Monitoring Committee (DMC), which is independent from the sponsor and competing interests, comprises clinical experts, trial experts, investigators, and a statistician and will conduct regular monitoring tests (approximately every 4 months) to ensure the authenticity of the data. A trial steering committee, comprising the $\mathrm{Pl}$, clinicians and researcher with experience in this area will meet every 6 months to review study progress and address any concerns subsequent to DMC reports. The study will be monitored on an approximate 6 monthly basis by the sponsor by a blinded and unblinded study monitor of extensive experience. These monitoring visits will ensure appropriate patient eligibility for the study, staff training, accurate recording of data including source data verification and protocol deviations. The study will be open to external auditing by the HPRA (a process independent from investigators and the sponsor), throughout the time-frame of this study to ensure adherence to GCP.

The end of trial will be the date of the last visit of the last participant (50th randomised participant). The sponsor, the funder (Stanley Medical Research Institute) and/or data safety and monitoring board/trial steering committee have the right at any time to terminate the study for clinical or administrative reasons.

This trial may be subject to internal or external auditing or inspection procedures to ensure adherence to GCP. Access to all trial-related documents will be given at that time. 


\section{Dissemination}

The results of this study will be published in peer-reviewed journals and be presented at domestic or international academic congresses. Individualised results will be reported by the researcher to the participants of this study, after data publication in peer-reviewed journals. Data will be available for public access via the National Institute for Mental Health Data Archive one year after submission of all participant data.

\section{Discussion}

The purpose of this study is to examine the efficacy and safety of IV scopolamine compared to placebo in individuals with bipolar disorder who are experiencing a depressive episode. In addition, this study will also assess whether IV scopolamine, compared to placebo, impacts cognition and functioning in individuals with bipolar disorder experiencing a depressive episode of at least moderate severity. This is the first study to our knowledge to investigate the antidepressant effects of IV scopolamine in an exclusively bipolar disorder cohort. This study can help further research investigate the potential antidepressant effect of anticholinergic medications.

Some commonly documented adverse effects of IV scopolamine include: drowsiness, blurred vision, dry mouth, thirst, and urinary retention. No serious adverse events are expected from the IV scopolamine infusion and no serious adverse events were witnessed in previous studies using IV scopolamine in depressed patients.

In relation to ethical considerations and patient confidentiality, the vulnerability of this study group is fully appreciated, and every effort will be undertaken to protect their safety and well-being. During their time on the trial, participants will continue usual care, as recommended by their responsible clinical team. In line with the applicable regulatory requirements, consenting processes (performed by trained clinical staff with GCP certification) will be standardised and a robust standard operating procedure (SOP) for consenting participants will be adhered to.

\section{Trial Status}

Recruitment has commenced in March 2021. The duration of this study is 3 years, or until the number of necessary participants who have completed randomisation $(n=50)$ have been attained.

\section{Abbreviations}

\section{$\mathrm{AE}$}

Adverse Event

ANCOVA

Analysis of Covariance

AUDIT

Alcohol Use Disorder Identification Test

CANTAB

Cambridge Neuropsychological Test Automated Battery

CGI-I

Clinical Global Impression-Improvement

CGI-S

Clinical Global Impression-Severity

CRFG

Clinical Research Facility, Galway

DMC

Data Monitoring Committee

DSM

Diagnostic Statistics Manual

ERT

Emotion Recognition Task

GAF

Global Assessment of Functioning

GCP

Good Clinical Practice

HDRS

Hamilton Depression Rating Scale

HPRA

Health Products Regulatory Authority

IV

Intravenous

MADRS

Montgomery and Asberg Depression Scale 
MDD

Major Depressive Disorder

NEO PI-FFI

NEO Personality Inventory-Five Factor Inventory

OTS

One Touch Stockings of Cambridge

PRISE

Patient Rated Inventory of Side Effects

PAL

Paired Associates Learning

POMS

Profile of Mood States

REC

Research Ethics Committee

RVP

Rapid Visual Information Processing

SAE

Serious Adverse Event

SCID-RV

Structured Clinical Interview for DSM

SOP

Standard Operating Procedure

SUSAR

Suspected Unexpected Serious Adverse Reaction

VAS

Visual Analogue Scale

WAIS

Wechsler Adult Intelligence Scale

YMRS

Young Mania Rating Scale

\section{Declarations}

\section{Ethical Approval}

This study was approved by the Galway University Hospitals Research Ethics Committee (Ref 88/12) on January $9^{\text {th }}, 2020$. Annual reports to the National Research Ethics Committee will be provided.

\section{Consent for Publication}

Consent for publication and dissemination of data was attained as part of ethical approval. Publication of study results in research journals and/or conferences is clearly outlined in the participant information leaflet.

\section{Availability of data and material}

Data will be available for public access via the National Institute for Mental Health Data Archive (Bethesda, Maryland, USA) one year after submission for participant data where informed written consent is provided.

\section{Competing interests}

The authors declare that they have no competing interests.

\section{Funding}

The SCOPE-BD trial is funded by the Stanley Research Medical Institute (17T-002).

\section{Authors' contributions}

$\mathrm{CM}$ and $\mathrm{BH}$ participated in the study design, contributed to the writing of the study protocol, drafting and editing of this manuscript. $\mathrm{BH}$ conceptualised the study and is the principal investigator of this study. $\mathrm{CMCD}$ and $\mathrm{DC}$ are co-applicants on the study grant application and members of the trial steering group and were involved in study protocol drafting. All authors have contributed to the writing, and have approved the final manuscript. 
We wish to acknowledge staff members of the Clinical Research Facility that have supported the set-up and commencement of this study. We wish to acknowledge clinicians who have supported recruitment for this RCT and participants who have agreed to participate in this study.

\section{Sponsor}

Vice-President for Research, National University of Ireland Galway,

E-Mail: vpresearch@nuigalway.ie

Tel: +353-91-495678

\section{References}

1. American Psychiatric A: Diagnostic and statistical manual of mental disorders: DSM-5. [DSM V], 5th edition edn. Washington, DC: American Psychiatric Publishing; 2013.

2. Ferrari AJ, Stockings E, Khoo JP, Erskine HE, Degenhardt L, Vos T, Whiteford HA. The prevalence and burden of bipolar disorder: findings from the Global Burden of Disease Study 2013. Bipolar Disord. 2016;18(5):440-50.

3. Fournier JC, DeRubeis RJ, Hollon SD, Dimidjian S, Amsterdam JD, Shelton RC, Fawcett J. Antidepressant drug effects and depression severity: a patientlevel meta-analysis. JAMA. 2010;303(1):47-53.

4. Yildiz A, Vieta E, Leucht S, Baldessarini RJ. Efficacy of antimanic treatments: meta-analysis of randomized, controlled trials. Neuropsychopharmacology. 2011;36(2):375-89.

5. Drevets WC, Furey ML. Replication of scopolamine's antidepressant efficacy in major depressive disorder: a randomized, placebo-controlled clinical trial. Biol Psychiatry. 2010;67(5):432-8.

6. Ellis JS, Zarate CA Jr, Luckenbaugh DA, Furey ML. Antidepressant treatment history as a predictor of response to scopolamine: clinical implications. J Affect Disord. 2014;162:39-42.

7. Furey ML, Drevets WC. Antidepressant efficacy of the antimuscarinic drug scopolamine: a randomized, placebo-controlled clinical trial. Arch Gen Psychiatry. 2006;63(10):1121-9.

8. Janowsky DS. Serendipity strikes again: scopolamine as an antidepressant agent in bipolar depressed patients. Curr Psychiatry Rep. 2011;13(6):443-5.

9. Furey ML, Khanna A, Hoffman EM, Drevets WC. Scopolamine produces larger antidepressant and antianxiety effects in women than in men. Neuropsychopharmacology. 2010;35(12):2479-88.

10. Furey ML, Nugent AC, Speer AM, Luckenbaugh DA, Hoffman EM, Frankel E, Drevets WC, Zarate CA. Jr.: Baseline mood-state measures as predictors of antidepressant response to scopolamine. Psychiatry Res. 2012;196(1):62-7.

11. Gillin JC, Sutton L, Ruiz C, Darko D, Golshan S, Risch SC, Janowsky D. The effects of scopolamine on sleep and mood in depressed patients with a history of alcoholism and a normal comparison group. Biol Psychiatry. 1991;30(2):157-69.

12. Newhouse PA, Sunderland T, Tariot PN, Weingartner H, Thompson K, Mellow AM, Cohen RM, Murphy DL. The effects of acute scopolamine in geriatric depression. Arch Gen Psychiatry. 1988;45(10):906-12.

13. Khajavi D, Farokhnia M, Modabbernia A, Ashrafi M, Abbasi SH, Tabrizi M, Akhondzadeh S. Oral scopolamine augmentation in moderate to severe major depressive disorder: a randomized, double-blind, placebo-controlled study. J Clin Psychiatry. 2012;73(11):1428-33.

14. Apfel CC, Zhang K, George E, Shi S, Jalota L, Hornuss C, Fero KE, Heidrich F, Pergolizzi JV, Cakmakkaya OS, et al. Transdermal scopolamine for the prevention of postoperative nausea and vomiting: a systematic review and meta-analysis. Clin Ther. 2010;32(12):1987-2002.

15. Stankovic AS, Alvarenga DL, Coleman Daniels VR, Simmons RG, Buckey JC, Putcha L. Intranasal Scopolamine for Motion Sickness. Aerosp Med Hum Perform. 2019;90(11):917-24.

16. Takeuchi I, Suzuki T, Kishi T, Kanamori D, Hanya M, Uno J, Fujita K, Kamei H. Effect of Scopolamine Butylbromide on Clozapine-induced Hypersalivation in Schizophrenic Patients: A Case Series. Clin Psychopharmacol Neurosci. 2015;13(1):109-12.

17. Bonner TI, Buckley NJ, Young AC, Brann MR. Identification of a family of muscarinic acetylcholine receptor genes. Science. 1987;237(4814):527-32.

18. Hulme EC, Birdsall NJ, Buckley NJ. Muscarinic receptor subtypes. Annu Rev Pharmacol Toxicol. 1990;30:633-73.

19. Davis KL, Berger PA, Hollister LE, Defraites E. Physostigmine in mania. Arch Gen Psychiatry. 1978;35(1):119-22.

20. Janowsky DS, el-Yousef MK, Davis JM, Sekerke HJ. A cholinergic-adrenergic hypothesis of mania and depression. Lancet. 1972;2(7778):632-5.

21. Nabulsi L, Farrell J, McPhilemy G, Kilmartin L, Dauvermann M, Akudjedu T, Najt P, Ambati S, Martyn F, McLoughlin J, et al: Normalization of Impaired Emotion Inhibition in Bipolar Disorder Mediated by Cholinergic Neurotransmission in the Cingulate Cortex. Neuropsychopharmacology.

22. Cannon DM, Carson RE, Nugent AC, Eckelman WC, Kiesewetter DO, Williams J, Rollis D, Drevets M, Gandhi S, Solorio G, et al. Reduced muscarinic type 2 receptor binding in subjects with bipolar disorder. Arch Gen Psychiatry. 2006;63(7):741-7.

23. Cannon DM, Klaver JK, Gandhi SK, Solorio G, Peck SA, Erickson K, Akula N, Savitz J, Eckelman WC, Furey ML, et al. Genetic variation in cholinergic muscarinic-2 receptor gene modulates M2 receptor binding in vivo and accounts for reduced binding in bipolar disorder. Mol Psychiatry. 2011;16(4):407-18.

24. Rosenkranz GK. Remarks on designs enriching for placebo non-responders. Clin Trials. 2016;13(3):338-43.

25. Babor TF. The Alcohol Use Disorders Identification Test: Guidelines for Use in Primary Health Care. Geneva: World Health Organization; 2001. 
26. Guy W: ECDEU assessment manual for psychopharmacology, Rev. 1976. edn. Rockville, Md: U.S. Dept. of Health, Education, and Welfare, Public Health Service, Alcohol, Drug Abuse, and Mental Health Administration, National Institute of Mental Health, Psychopharmacology Research Branch, Division of Extramural Research Programs; 1976.

27. Heatherton TF, Kozlowski LT, Frecker RC, Fagerstrom KO. The Fagerstrom Test for Nicotine Dependence: a revision of the Fagerstrom Tolerance Questionnaire. Br J Addict. 1991;86(9):1119-27.

28. Hall RC. Global assessment of functioning. A modified scale. Psychosomatics. 1995;36(3):267-75.

29. Hamilton M. A rating scale for depression. J Neurol Neurosurg Psychiatry. 1960;23:56-62.

30. Montgomery SA, Asberg M. A new depression scale designed to be sensitive to change. Br J Psychiatry. 1979;134:382-9.

31. Costa PT, McCrae RR, Psychological Assessment Resources I: Revised NEO Personality Inventory (NEO PI-R) and NEO Five-Factor Inventory (NEO-FFI). Odessa, Fla: Psychological Assessment Resources; 1992.

32. Rush AJ, Fava M, Wisniewski SR, Lavori PW, Trivedi MH, Sackeim HA, Thase ME, Nierenberg AA, Quitkin FM, Kashner TM, et al. Sequenced treatment alternatives to relieve depression (STAR* D): rationale and design. Control Clin Trials. 2004;25(1):119-42.

33. McNair DM, Lorr M, Droppleman LF. Manual for the profile of mood states. San Diego: Educational \& Industrial Testing Service; 1971.

34. First MB, Williams JBW, Karg RS, Spitzer RL, American Psychiatric Association P, American Psychiatric A. SCID-5-RV: structured clinical interview for DSM-5 disorders - research version. Arlington: American Psychiatric Association Publishing; 2015.

35. Young RC, Biggs JT, Ziegler VE, Meyer DA. A rating scale for mania: reliability, validity and sensitivity. Br J Psychiatry. 1978;133:429-35.

36. Robbins TW, James M, Owen AM, Sahakian BJ, Mclnnes L, Rabbitt P. Cambridge Neuropsychological Test Automated Battery (CANTAB): a factor analytic study of a large sample of normal elderly volunteers. Dement Geriatr Cogn Disord. 1994;5(5):266-81.

37. Wechsler D. Manual for the Wechsler adult intelligence scale. New York: Psychological Corporation; 1955.

38. ICH Good. Clinical Practice E6 (R2) https://globalhealthtrainingcentre.tghn.org/ich-good-clinical-practice/.

\section{Figures}

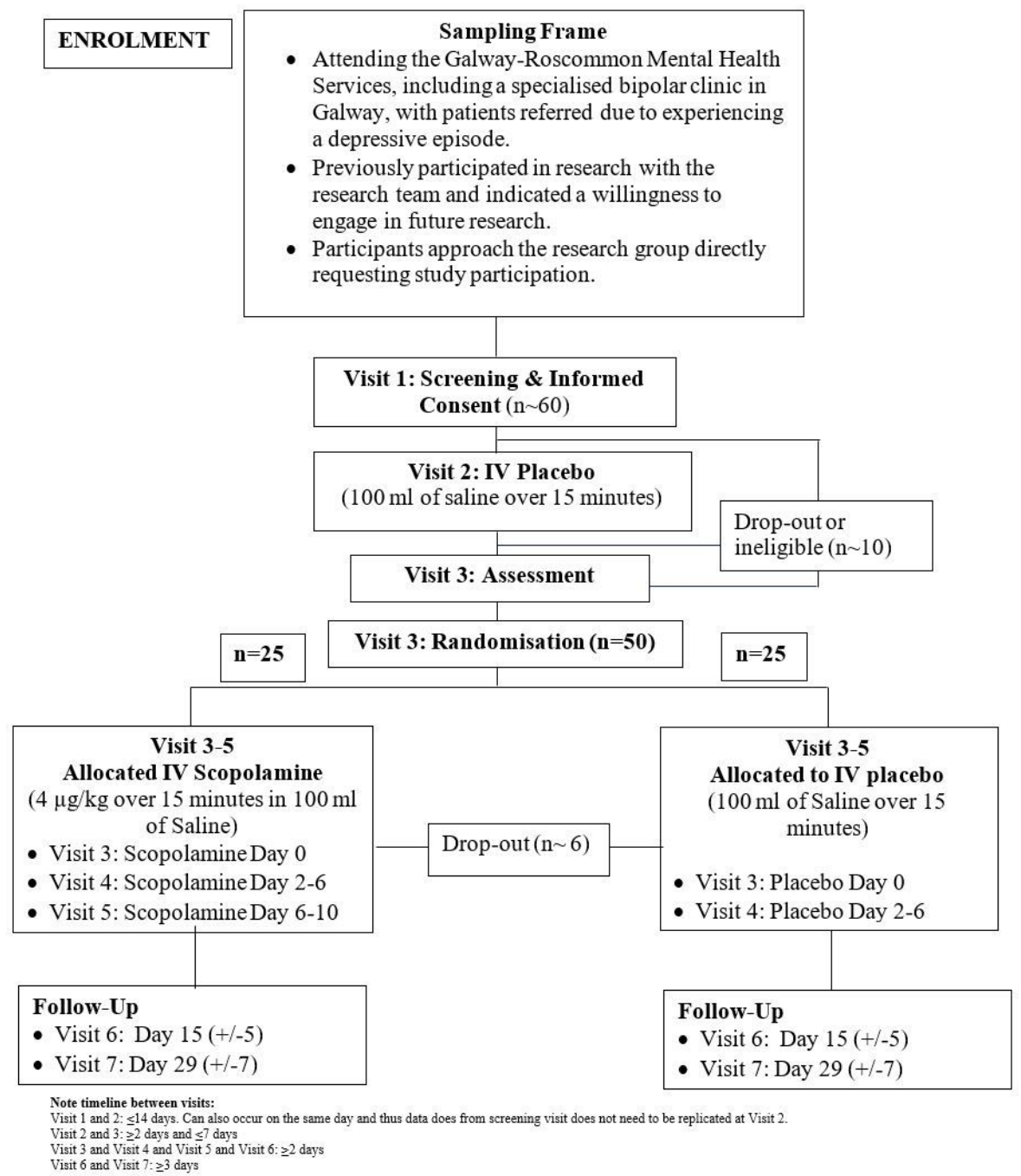

Figure 1

Page $11 / 12$ 
Study Design

\section{Supplementary Files}

This is a list of supplementary files associated with this preprint. Click to download.

- SPIRITchecklistCMFinal.doc 